\title{
Depletion of polycomb repressive complex 2 core component EED impairs fetal hematopoiesis
}

\author{
Wenhua Yu ${ }^{1,4}$, Fang Zhang ${ }^{1,4}$, Shiyan Wang ${ }^{1}$, Yi Fu', Jiahuan Chen ${ }^{1}$, Xiaodong Liang ${ }^{1}$, Huangying Le ${ }^{1}$, William T Pu ${ }^{\star 2,2}$ and \\ Bing Zhang ${ }^{\star, 1}$
}

Polycomb repressive complex 2 (PRC2), a H3K27me3 methyltransferase complex, promotes the development of many organs by silencing ectopic transcription program. However, currently little is known about the role of PRC2 in blood and vascular development. In this study, we interrogated the function of embryonic ectoderm development (EED), a core PRC2 component, in both endothelial and hematopoietic tissues by inactivating a floxed murine EED allele with Tie2Cre, which catalyzes recombination in endothelial and hematopoietic lineages. Murine EED ${ }^{\text {fl/fil }}$;Tie2Cre $\left(E E D^{\mathrm{CKO}}\right.$ ) embryos died at embryonic day (E) 13.5. We did not observe structural abnormalities of blood vessels or cardiac valves, suggesting that EED is dispensable in endothelial cells for initial steps of vascular development. EED ${ }^{\mathrm{CKO}}$ embryos were pale and had abnormal livers. Flow cytometry of fetal liver cells showed that EED depletion significantly impeded erythroid maturation. There was a corresponding increase in myeloid progenitors and granulocytes and macrophages, suggesting an attenuated differentiation path in myeloid lineages. Moreover, EED depletion impaired the generation of hematopoietic stem cells. Collectively, our study demonstrates that within Tie2Crerecombined embryonic cells, EED is required for proper erythropoiesis and for formation of hematopoietic progenitor and stem cells, but is dispensable for endothelial lineage commitment and early vascular patterning.

Cell Death and Disease (2017) 8, e2744; doi:10.1038/cddis.2017.163; published online 13 April 2017

Polycomb repressive complex 2 (PRC2) represses gene transcription by trimethylating histone $\mathrm{H} 3$ on lysine 27 (H3K27me3). The core PRC2 complex is comprised of embryonic ectoderm development (EED), Suppressor of Zeste12 homolog (SUZ12), retinoblastoma-associated protein 48 (RbAp48), and a catalytic subunit, either Enhancer of Zeste Homolog 1 or 2 (EZH1 or EZH2). ${ }^{1,2} \mathrm{EZH} 1$ and EZH2 are mutually exclusive in the same PRC2 complex and are partially redundant at certain developmental and disease stages., ${ }^{3,4}$ EED is a core component of PRC2 complex that interacts with $E Z H 1$ or $E Z H 2$ through a WD40 domain. In addition, EED recognizes $\mathrm{H} 3 \mathrm{~K} 27 \mathrm{me} 3$ through an aromatic cage structure to recruit PRC2 to established H3K27me3 and thereby enforce maintenance of this repressive epigenetic mark. ${ }^{5,6}$

PRC2 has essential roles of regulating cell fate commitment, organogenesis, homeostasis and disease-related tissue remodeling. ${ }^{1}$ In early developing mouse embryos, homozygous Eed null mutation abolished global H3K27 methylation and caused defects in primitive streak formation and fetal lethality at E9.5. ${ }^{7,8} \mathrm{EZH} 2$ also controls the fate of multiple types of tissue progenitors. ${ }^{9}$ Depletion of EZH2 in epidermal precursor cells promoted premature epidermal differentiation and barrier acquisition. ${ }^{10,11} \mathrm{EZH} 2$ also had developmental-stage-specific roles in regulating cardiomyocyte differentiation and proliferation. ${ }^{12,13}$ In adult vasculature,
EZH2 was required for VEGF-induced silencing of vasohibin1 (VASH1), which promoted angiogenesis in ovarian cancer. ${ }^{14}$

Both endothelial cell (EC) and hematopoietic cells originate from a common progenitor, the hemangioblast. Hemogenic ECs within the yolk sac give rise to the first population of hematopoietic stem cells (HSCs), which migrate to aortagonad mesonephros (AGM) and then to the fetal liver, which then becomes the main site of fetal hematopoiesis. Fetal liver HSCs establish the common myeloid progenitor (CMP) and the common lymphoid progenitor (CLP), multipotent progenitors that differentiate into the major blood lineages. CMPs further differentiate into megakaryocyte-erythrocyte progenitors (MEPs), precursors of erythrocytes and megakaryocytes, and granulocyte-macrophage progenitors (GMPs), precursors of granulocytes and monocyte/macrophages. ${ }^{15-17}$

Functional links between PRC2, hematopoiesis, and hematopoietic malignancies have been recently unveiled. Human genetic studies revealed that mutations affecting $E Z H 2, E E D$, SUZ12, and JARID2, encoding PRC2 subunits and associated proteins, cause hematopoietic diseases including myelodysplastic syndrome, T-cell acute lymphoblastic leukemia, and B cell lymphoma. ${ }^{18-21}$ However, the functions of PRC2 in hematopoietic development are currently less clear. Conditional deletion of Ezh2 in hematopoietic and vascular lineages of mouse embryos by Tie2Cre was lethal at mid-gestation due to insufficient expansion of hematopoietic

\footnotetext{
${ }^{1}$ Key Laboratory of Systems Biomedicine, Shanghai Center for Systems Biomedicine, Shanghai Jiao Tong University, Shanghai 200240, China; ${ }^{2}$ Department of Cardiology, Boston Children's Hospital, Boston, MA 02115, USA and ${ }^{3}$ Harvard Stem Cell Institute, Cambridge, MA 02138, USA

*Corresponding author: WT Pu, Department of Cardiology, Boston Children's Hospital, 300 Longwood Avenue, Boston, MA 02115, USA. Tel: 617 919 2091; E-mail: wpu@pulab.org

or B Zhang, Shanghai Center for Systems Biomedicine, Shanghai Jiao Tong University, Shanghai 200240, China. Tel: 862134208691 ; Fax: +862 134 206059;

E-mail: bingzhang@sjtu.edu.cn

${ }^{4}$ These authors contributed equally to this work.

Received 18.1.17; revised 13.3.17; accepted 14.3.17; Edited by H-U Simon
} 
stem/progenitor cells (HSCs). ${ }^{22}$ In contrast, conditional deletion of EED in HSCs by Vav ${ }^{\text {Cre }}$ did not significantly influence fetal HSC hemostasis but became significant in the adulthood. ${ }^{23}$ Thus, the role of PRC2 and its component subunits in blood and vessel development require elucidation.

In this study, we used Tie2Cre to inactivate EED in developing blood and vascular lineages. Because there is no functional redundant ortholog of EED, this should abolish PRC2 function in these lineages and circumvent functional redundancy between $E Z H 1$ and $E Z H 2$. We found that EED is dispensable for EC specification and initial development into vessels and cardiac valve precursors, but is essential for normal population of the fetal liver by HSCs and for erythropoiesis. Combined with previous studies on PRC2, our results illustrated a tissue-context-dependent and developmental-stage-specific role of $\mathrm{PRC2}$ in controlling hematopoiesis.

\section{Results}

EED is required in the Tie2Cre lineage for normal embryonic development. EED ${ }^{\text {flox }}$ mice were described previously and develop normally. ${ }^{23}$ Although heterozygosity for a null Eed allele was previously reported to cause myeloand lympohoproliferative defects, ${ }^{24}$ Tie2Cre;EED ${ }^{\text {fl/wt }}$ mice survived normally to adulthood and were fertile (Table 1). However, no live Tie2Cre;EED ${ }^{\text {fl/fl }}$ (EED conditional knockout, abbreviated as EED ${ }^{\mathrm{CKO}}$ ) mice were observed at birth (Table 1), indicating that EED is required in the Tie2Cre lineage for normal embryonic development.

To determine the time of embryonic lethality, we analyzed litters at E11.5 and E13.5. At E11.5, EED ${ }^{\mathrm{CKO}}$ were present at the expected Mendelian frequency, whereas $70 \%$ of mutant embryos were no longer viable at E13.5 (Table 1, Figures 1a and b). However, E11.5 EED ${ }^{\mathrm{CKO}}$ embryos already showed developmental abnormalities, as they lacked large bloodperfused vessels including vitelline veins in the yolk sac (Figures 1c and d, black arrow) and primary head veins of the embryo (Figures 1c and d, yellow arrow). The region occupied by the liver was also notably pale (Figures 1c and d, asterisk).

EED deletion did not significantly alter the vascular patterning. Given that EED is an essential component of PRC2, which deposits H3K27me3, we performed immunostaining to measure bulk H3K27me3 levels. Immunofluorescent staining demonstrated substantially weaker H3K27me3 signal in endothelial cells (Figures $2 a$ and $b$ ), consistent with efficient EED deletion in these cells by Tie2Cre. This also suggested the possibility that the observed absence of blood-

Table 1 Survival rate of $\mathrm{Tie} 2^{\mathrm{Cre}} ; \mathrm{EED}^{\mathrm{fl} / \mathrm{fl}}$ knockout mouse

\begin{tabular}{lcccc}
\hline Age & EED $^{\text {fl/wt }}$ & EED $^{\mathrm{fl} / \mathrm{fl}}$ & TieCre $^{+} E E D^{\mathrm{fl} / \mathrm{wt}}$ & TieCre $^{+} \mathrm{EED}^{\mathrm{fl} / \mathrm{fl}}$ \\
\hline P0 & $16(33 \%)$ & $16(33 \%)$ & $16(33 \%)$ & $0(0 \%)$ \\
E13.5 & $16(24 \%)$ & $20(29 \%)$ & $18(26 \%)$ & $14(4 \mathrm{viable}=6 \%)$ \\
E11.5 & $30(30 \%)$ & $27(27 \%)$ & $20(20 \%)$ & $23(23 \%)$ \\
\hline
\end{tabular}

Table indicates number and (\%) of embryos found at each stage. filled vessels in EED ${ }^{\text {CKO }}$ embryos was due to abnormal vascular development (Figures $2 \mathrm{a}$ and $\mathrm{b}$ ).

To test this possibility, we examined blood vessels of wild-type and EED ${ }^{\mathrm{CKO}}$ embryos at E9.5 and E12.5 by PECAM1 whole-mount staining, a marker of vascular endothelial cell. Unexpectedly, at E9.5, there was no global angiodysplasia observed in EED mutants. The large vessels such as the pharyngeal arch arteries, midline dorsa aorta, cardinal vein, intersomitic vessels, and large cerebral vessels were well developed in EED ${ }^{\mathrm{CKO}}$ (Figures $2 \mathrm{c}$ and d). The small vessels formed complete vascular plexuses with similar density and pattern to control littermates. We also checked the vasculature of E12.5 embryos and found no significant difference in vascular density or pattern in EED ${ }^{\mathrm{CKO}}$ compared with control (Figures $2 e$ and $f$ ).

Endothelial cells of the heart contribute to heart valve mesenchyme formation through endothelial-to-mesenchymal transition, and Tie2Cre-driven mutations in EC genes have caused lethal heart valve defects. ${ }^{25,26}$ To test whether the deletion of EED results in valve hypoplasia, we examined heart valves in E12.5 EED ${ }^{\mathrm{CKO}}$ and control littermates. Cardiac valve size and morphology were comparable between mutant and wild-type embryos (Figures $2 g$ and $h$ ). Taken together, these data suggest that EED depletion did not significantly influence vessel or valve formation, and therefore are unlikely to account for the observed embryonic lethality.

Deletion of EED attenuated the myeloid lineages commitment. Tie2Cre drives recombination in almost all hematopoietic cells, in addition to endothelial cells. ${ }^{27}$ As the deletion of PRC2 has been reported to induce defective hematopoiesis, ${ }^{23,24}$ we hypothesized that death of EED ${ }^{\text {CKO }}$ embryos is due to the abnormal hematopoiesis. Between $\mathrm{E} 11$ and $\mathrm{E} 15.5$, the fetal liver is the primary site of hematopoiesis. ${ }^{15,28}$ Given the abnormal appearance of the fetal liver on gross embryo inspection (Figures 1c and d), we examined fetal liver morphology on H\&E-stained histological sections and found that EED ${ }^{\mathrm{CKO}}$ liver size was significantly reduced (Figures $3 a$ and b). Hepatic structure was poorly organized, and the cytoplasm of blood cells within the liver were swollen and more eosinophilic than controls (Figure 3b). All together, these data suggested disrupted fetal hematopoiesis.

To check the status of hematopoiesis, especially erythropoiesis, whose deficiency could have contributed to the pale appearance of EED ${ }^{\mathrm{CKO}}$ embryos, we isolated hematopoietic cells from fetal liver and examined their lineage composition by flow cytometry. Using a Cre-activated Rosa26-floxed-stopYFP (Rosa26 ${ }^{\text {tSYFP }}$ ) reporter, we determined that over $90 \%$ of hematopoietic cells were $\mathrm{YFP}^{+}$(Figures $4 \mathrm{a}$ and b), confirming efficient Tie2Cre-mediated recombination within the hematopoietic lineage. ${ }^{27} \mathrm{We}$ then evaluated erythropoiesis using flow cytometry to measure lineage differentiation markers. CD71 ${ }^{+}$ Ter $119^{+}$cells, representing more mature erythroblasts, were dramatically depleted compared with control $(9.4 \%$ versus $43 \%, P=0.0004$; Figures $4 c-e)$. In contrast, less mature $\mathrm{CD} 71^{+}$Ter $119^{-}$erythroblasts were significantly increased ( $18.6 \%$ versus $8.1 \%, P=0.0001$, Figures $4 \mathrm{c}$, $d$ and $\mathrm{f}$ ), which suggested that EED is required for erythroblast maturation.

Erythroblasts arise from a common myeloid progenitor (CMP), which also differentiates into the granulocyte- 

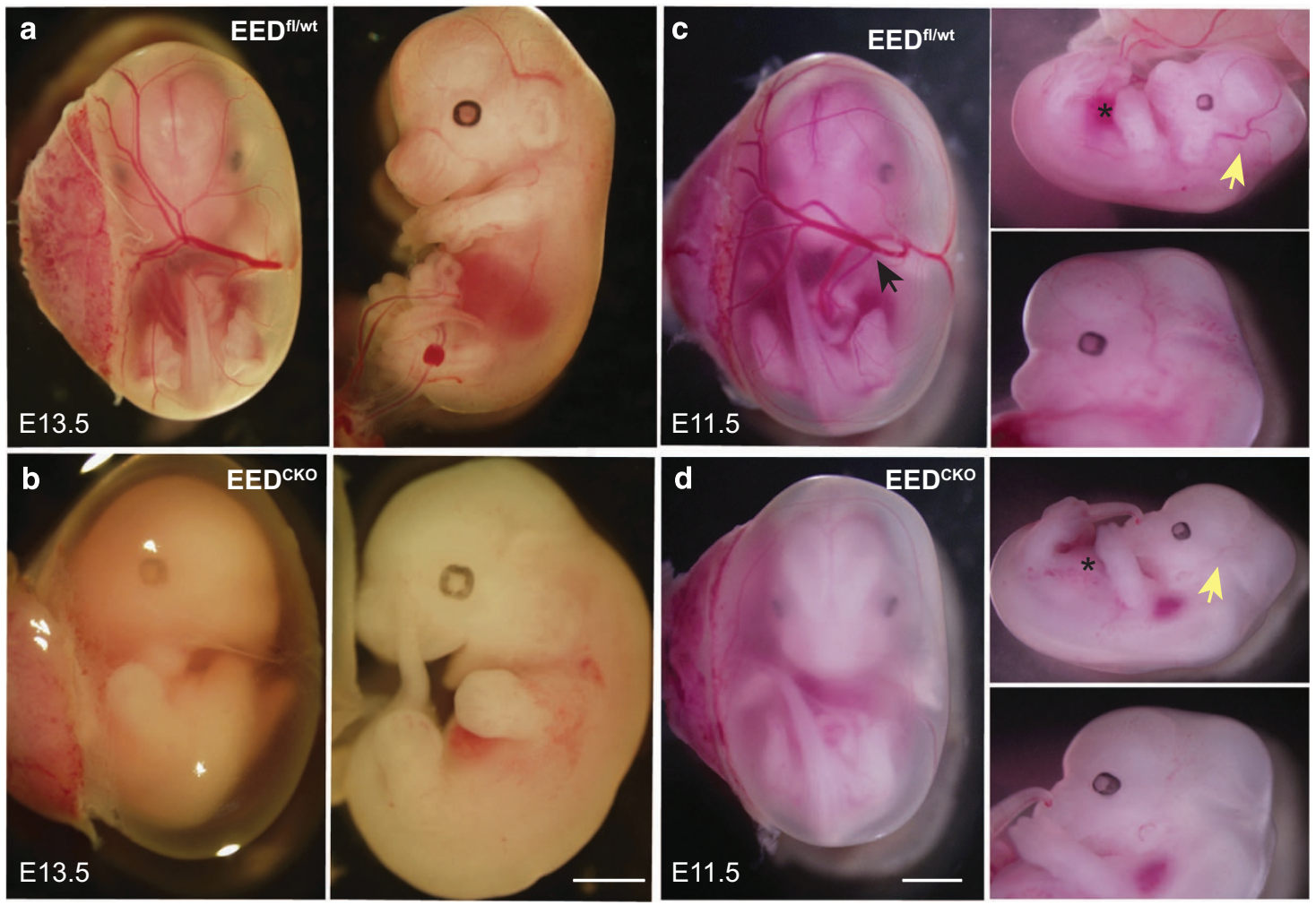

Figure $1 \mathrm{EED}^{\mathrm{CKO}}$ mouse is embryonic lethal at mid-gestation. (a and $\mathbf{b}$ ) Images of wild-type and EED ${ }^{\mathrm{CKO}}$ embryos at E13.5. Most EED ${ }^{\mathrm{CKO}}$ embryos had died by this stage. (c and d) Images of wild-type and EED ${ }^{\mathrm{CKO}}$ embryos at E11.5. EED ${ }^{\mathrm{CKO}}$ embryos show significant paleness and absence of blood-perfused vasculature. Asterisks overlie the fetal liver. Black arrow points to a vitelline vessel. Yellow arrow indicates primary head vein. Bar, $0.2 \mathrm{~cm}$ (b) or $0.3 \mathrm{~cm}$ (d)

macrophage progenitor (GMP), which subsequently differentiates into granulocytes and macrophages (GMC). ${ }^{17,29}$ The granulocytes and macrophages express the cell surface marker Mac1 and lack Ter119. ${ }^{17}$ By flow cytometry, MAC1 ${ }^{+}-$ Ter119 ${ }^{-}$cells increased almost 2.4-fold $(P=0.0001)$ in EED ${ }^{\text {CKO }}$ (Figures $4 \mathrm{~g}-\mathrm{i}$ ). This result suggests that EED depletion biases CMP differentiation towards GMC at the expense of the myeloid lineage.

EED depletion exhausted the HSC pool. Erythroblasts and granulocytes are derived from myeloid progenitors. To investigate whether EED is required for normal formation of myeloid progenitor cells, we measured multipotent hematopoietic and myeloid progenitors by flow cytometry. Myeloid progenitor cell (MPC) lacks canonical erythrocyte maker of CD71 and Ter119. Thus, we first isolated the CD71 ${ }^{-}$Ter119blood cells and further stained them with typical progenitor markers of Sca1 and cKit to gate the MPC (Figures 5a-d). The percentage of $\mathrm{CD} 71^{-} \mathrm{Ter} 119^{-} \mathrm{CKit}{ }^{+} \mathrm{Sca} 1^{-}$myeloid progenitors was increased by 2.5 -fold in EED ${ }^{\mathrm{CKO}}$ compared with control littermates $(P=0.0076$; Figure 5e), indicating EED depletion hindered MPC differentiation or promotes its proliferation or self-renewal. The total number of CD71- ${ }^{-}$Ter $119^{-} \mathrm{Sca}^{+}{ }^{+} \mathrm{CKit}^{+}$ (LSK) cells was not altered in EED ${ }^{\mathrm{CKO}}$ although they were significantly depleted in $\mathrm{C}^{-} 1^{-} \mathrm{Ter} 119^{-}$population $(P=0.1467$; Figure 5f).

The depletion of $\mathrm{cKit}^{+}$cells in CD71-Ter119- population indicates the loss of HSC in EED ${ }^{\text {CKO }}$ mouse. HSCs are hematopoiesis-committed multipotent cells that self-renew and give rise to all hematopoietic lineages. To test whether PRC2 regulates HSC generation or homeostasis, we stained for cell surface markers C48 and C150. Within $\mathrm{Lin}^{-} \mathrm{CD} 48^{-}$ $\mathrm{CD} 150^{+}$cells, HSC cells are the subset that expresses the pan-stem cell marker Sca ${ }^{23}$ (Figures 6a-d). Consistent with a previous study showing the impaired expansion of HSC cells following EZH2 depletion, the HSC population in EED ${ }^{\mathrm{KO}}$ was significantly reduced $(0.025$ versus $0.0056 \%, P=0.011$; Figure 6e). Taken together, these data indicate that EED deletion impaired the generation or maintenance of HSCs.

\section{Discussion}

The PRC2 complex is a master regulator of differentiation programs and organogenesis. In this study, we depleted EED, a core component of PRC2. Unlike EZH1 or EZH2, there is no known functional redundancy for EED within PRC2, and therefore EED inactivation should delineate required PRC2 functions. Inactivation of EED flox using Tie2Cre illustrated that EED depletion did not strongly affect vascular development but significantly disrupted erythroid maturation as well as HSC formation, homeostasis, and differentiation, which together led to embryonic lethality. These findings reinforced current knowledge of the essential role of PRC2 in regulating cell differentiation and homeostasis, while unveiling new roles for PRC2 in regulating hematopoiesis.

PRC2 and hematopoiesis. PRC2 has been recently shown to regulate the development and malignancy of multiple 

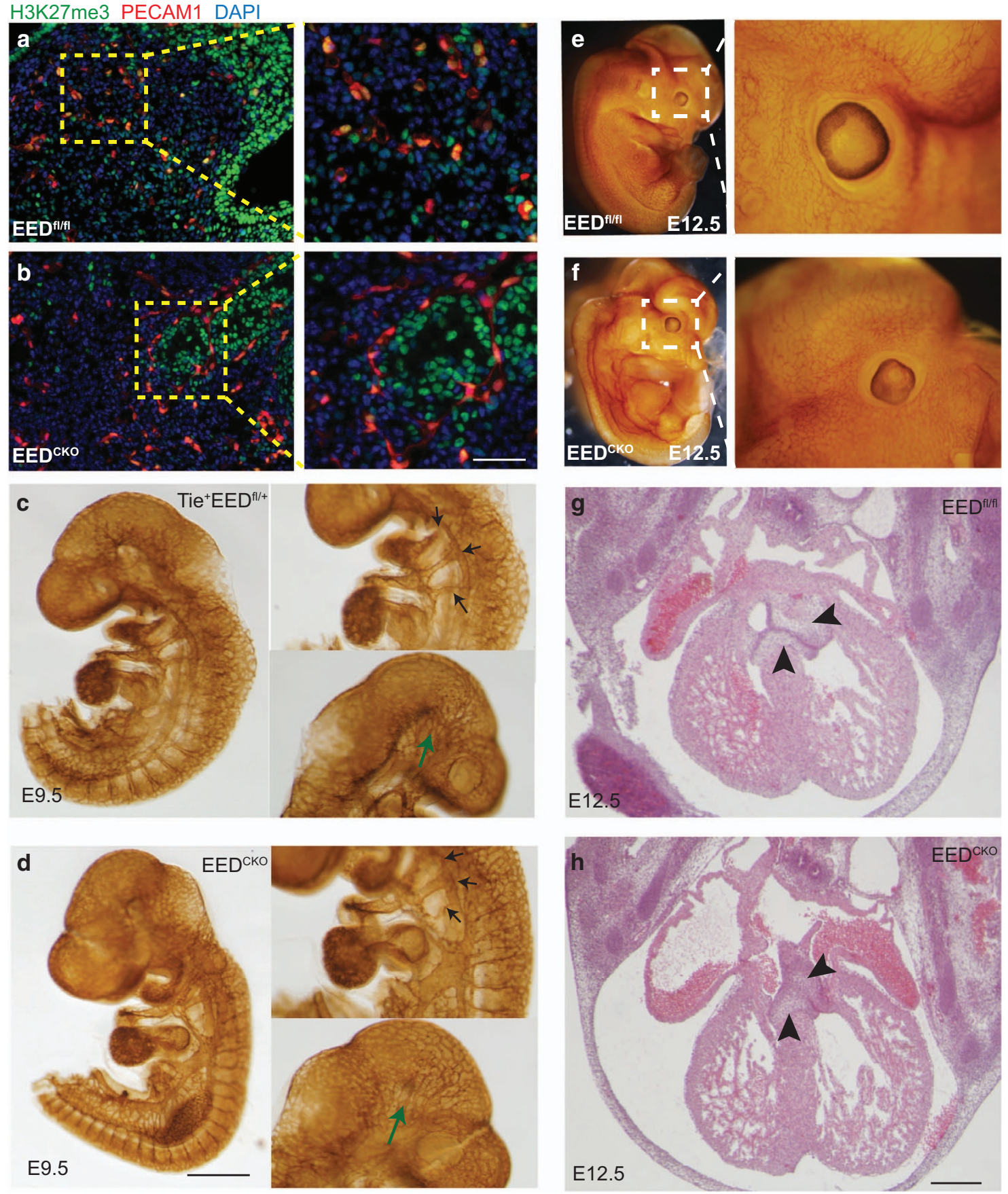

Figure 2 Normal blood vessel and cardiac valve development in EED ${ }^{\mathrm{CKO}}$. (a and b) Immunofluorescent images of skin tissue from E12.5 stage embryos. Overall, H3K27me3 signal was reduced in endothelial cells. Bar, $0.1 \mathrm{~cm}$. (c and d) PECAM1 whole-mount staining of E9.5 embryos. Black arrows point at branchial arch arteries. Green arrows point at cerebral vascular plexus. Bar, $0.1 \mathrm{~cm}$. (e and f) PECAM1 whole-mount staining of E12.5 embryos. ( $\mathbf{g}$ and $\mathbf{h}$ ) H\&E staining of E12.5 embryonic heart section. Arrowheads point at atrioventricular endocardial cushion. Bar, $50 \mu \mathrm{m}$

hematopoietic lineages, ${ }^{20,22,23,30,31}$ but its roles in these processes are still imprecisely defined. Ectopic expression of EZH2 in bone marrow HSCs promoted HSC proliferation and myeloproliferative disease. ${ }^{32}$ Conversely, inactivating mutations of PRC2 core members SUZ12 and EED, induced by $N$-ethyl- $N$-nitrosourea (ENU), disrupted HSC regeneration. ${ }^{30}$ Depletion of EZH2 by Tie2Cre caused defective HSC development in the fetal liver, but did not compromise adult
HSC capacity to reconstitute the bone marrow. ${ }^{22}$ However, a recent study reported that deletion of EED by $\mathrm{Vav}^{\mathrm{Cre}}$, which is active in hematopoietic cells, was compatible with fetal survival, leading to the opposite conclusion that PRC2 is dispensable for fetal HSC differentiation and maintenance. ${ }^{23}$ Results from these EZH2 and EED knockout studies have been difficult to compare due to the different Cre drivers, functional redundancy of EZH1/2, and non-canonical roles of 

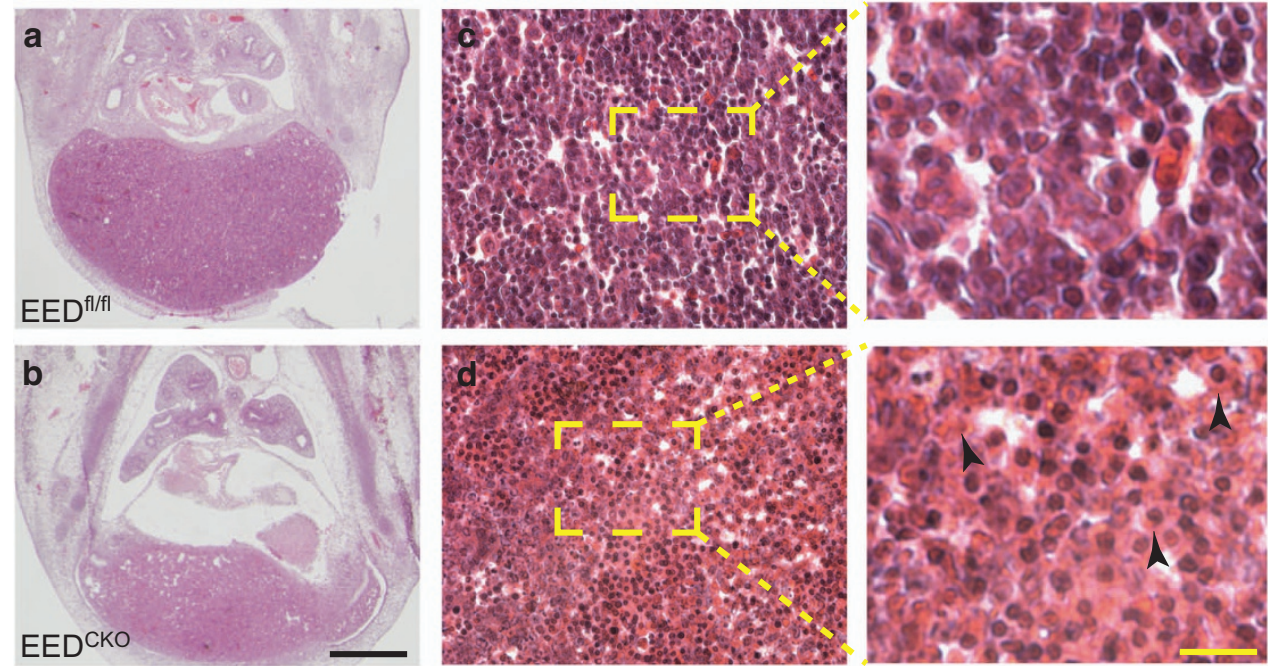

Figure 3 Hepatic abnormalities of EED ${ }^{\mathrm{CKO}}$ embryos. (a and $\left.\mathbf{b}\right) \mathrm{H} \& \mathrm{E}$ staining of E12.5 histological section through the liver. Arrowheads indicate swollen cells with eosinophilic cytoplasm. Black bar, $50 \mu \mathrm{m}$. Yellow bar, $10 \mu \mathrm{m}$
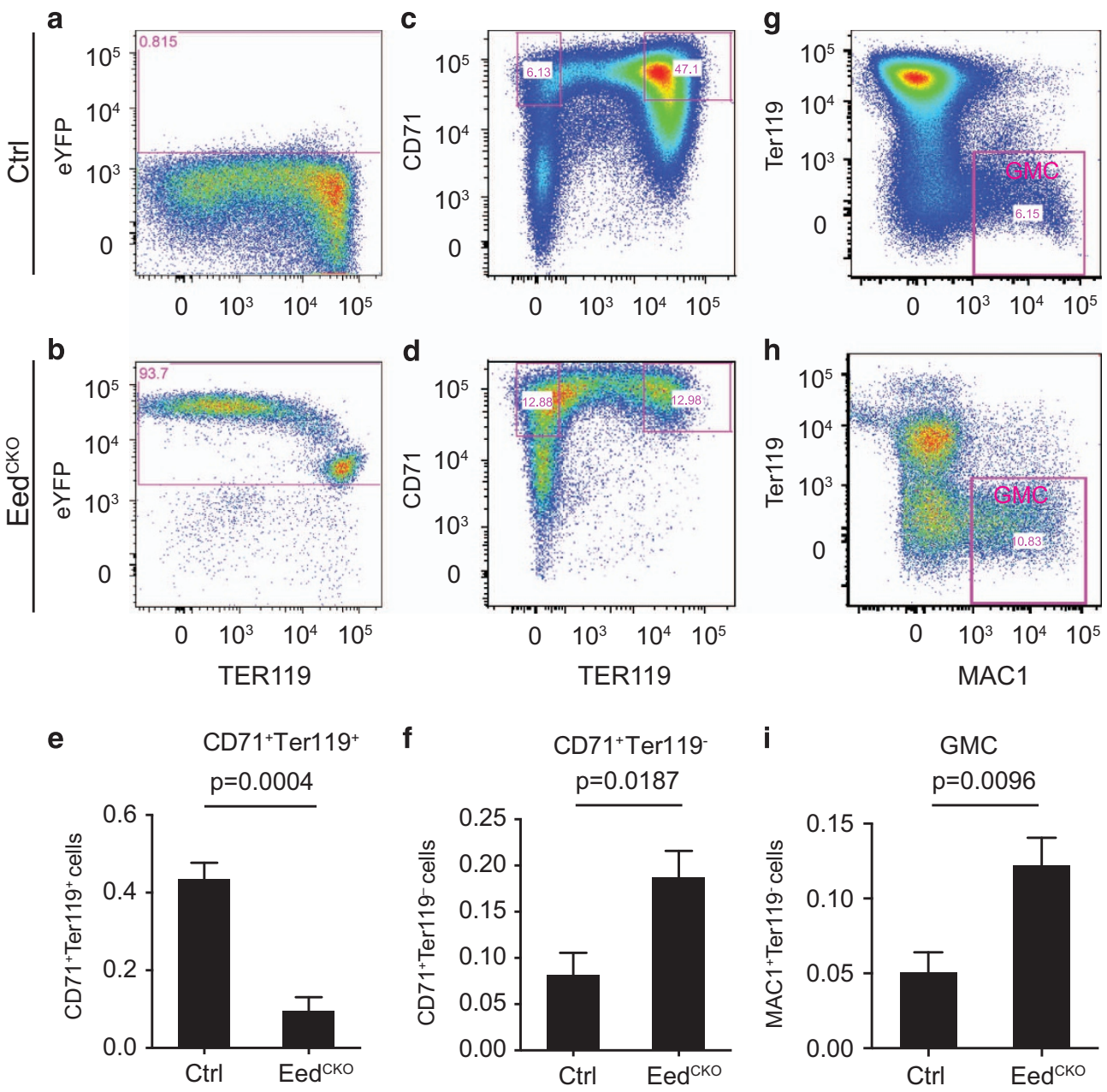

Figure 4 EED ${ }^{\mathrm{CKO}}$ caused defective erythrocyte maturation. Flow cytometry analysis of hematopoietic cells from hepatic liver. (a and $\left.\mathbf{b}\right)$ Detection of hematopoietic cells that expressed YFP from Tie2Cre-recombined Rosa26 $6^{\text {fsYFP }}$. (c and d) Flow cytometry analysis of erythroblasts $\left(\mathrm{CD} 71^{+}\right.$Ter $\left.119^{+}\right)$. (e) Quantitative analysis of $\mathrm{C} 71^{+}$Ter $119^{+}$ erythroblasts. Unpaired $t$-test, $n=4-7$. (f and $\mathbf{g}$ ) Flow cytometry analysis of less mature erythroblasts $\left(\mathrm{CD} 71^{+}{ }^{\top}\right.$ Ter $\left.119^{-}\right)$. (h) Quantitative analysis of $\mathrm{CD}^{-} 1^{+}$Ter $119^{-}$erythroblasts. Unpaired $t$-test, $n=4-7$. (i and j) Flow cytometry analysis of the granulocyte lineage (MAC1 ${ }^{+}$Ter119-). (k) Quantitative analysis of granulocyte lineage. Unpaired $t$-test, $n=4-7$ 
a

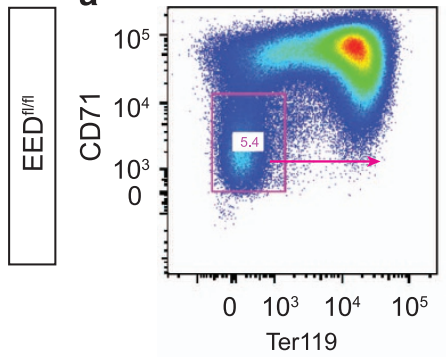

b

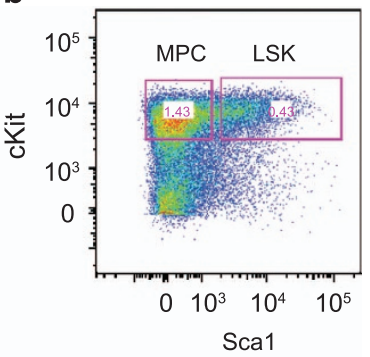

C

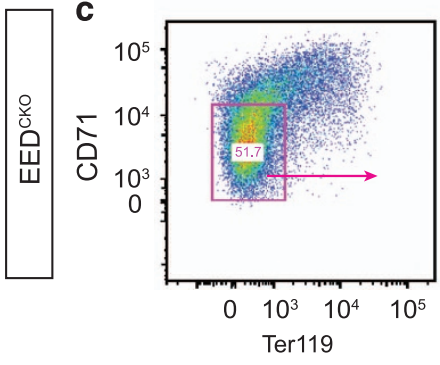

d

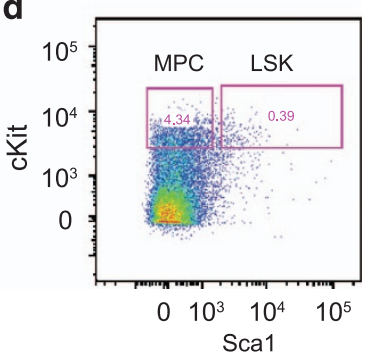

e

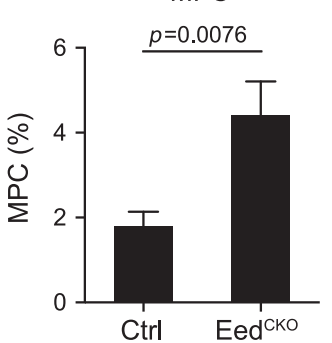

f

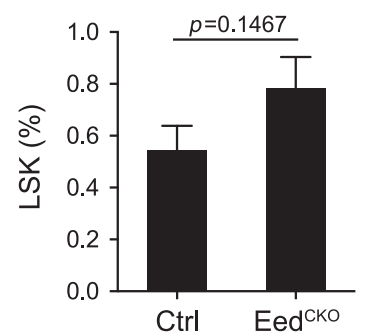

Figure 5 Eed deletion resulted in defective development of myeloid progenitor cells. (a-d) Flow cytometry analysis of myeloid progenitor cells (MPCs) and LSK cells from control and EEDCKO fetal liver. (e) Quantitative analysis of MPCs. Unpaired $t$-test, $n=3-6$. (f) Quantitative analysis of LSK cells. Unpaired $t$-test, $n=3-6$

EZH2 that are not dependent on EED. ${ }^{33}$ Furthermore, a recent study reported that EED functions as a scaffold protein that interacts with catalytic components of the PRC1 complex, ${ }^{34}$ adding further complexity to comparisons between EZH2 and EED knockout studies.

Our study reconciles the results of the Tie2Cre;Ezh2 $2^{\mathrm{fl} / \mathrm{fl}}$ and $\mathrm{Vav}^{\mathrm{Cre}} ; \mathrm{EED}^{\mathrm{f} / \mathrm{fl}}$ studies to a large extent by deleting EED ${ }^{\mathrm{f} / \mathrm{fl}}$ with Tie2Cre. We found that EED depletion by Tie2Cre disrupted HSC homeostasis and severely impaired erythropoiesis. We reason the different results from two experiments might be due to the earlier onset of Tie2Cre deletion in hemangioblasts. EEDdeficient hemangioblasts might be defective in maitenance or further differentiation, whereas this defect may not be exposed when EED ablation occurs in HSCs or their descendants. Although lethal effects of EED depletion on hematopoiesis may require its inactivation in angioblasts, the functional defect may not occur in angioblasts, as we have previously observed that altering PRC2 in progenitors may not have functional consequences until a later developmental time point, ${ }^{12}$ perhaps reflecting a 'memory' effect of the chromatin landscape.

Tie2Cre also efficiently targets endothelial cells, which contribute to form a stem cell niche to nurse multiple types of hemotapoietic progenitors including HSC by paracrine

mechanisms. ${ }^{15,35}$ Hence, even though we did not observe vascular abnormalities in the EED ${ }^{\mathrm{CKO}}$ mutants, it is possible that Tie2Cre-mediated EED deletion in EC could influence this paracrine mechanism and thereby cell non-autonomously influence HSC maintenance and differentiation. To test this hypothesis, a more EC-restricted Cre such as $\mathrm{CDH} 5^{\mathrm{CreERT} 23}$ will need to be used. ${ }^{36}$

PRC2 and vascular development. Recently Tie2Cre; $\mathrm{EZH} 2^{\mathrm{fl} / \mathrm{fl}}$ mice were reported to have defective vascular integrity and severe hemorrhage evident at E12.5 and due to the activation of $M m p 9 .{ }^{37}$ In our study, we did not find significant hemorrhage up to E12.5, a day before death. Thus, it is less likely that embryonic lethality of our Tie2Cre; $E E D^{f / / f l}$ mutants was due to vascular leakage. The divergent results might result from technical differences in mouse strain background or kinetics of Cre inactivation. An alternative intriguing possibility is that the more severe vascular phenotype of $\mathrm{EZH} 2^{\mathrm{f} / \mathrm{fl}}$ mutants reflects a non-canonical, EED-independent role of EZH2 in regulating endothelial cell genes such as Mmp9. Another potential contributor to the divergent results is the recently reported role of EED in PRC1 activity. ${ }^{34}$

In adult mice, siRNA-mediated Ezh2 knockdown was used to show that EZH2 promotes tumor angiogenesis by repressing anti-angiogenic factor Vash $1 .{ }^{14}$ We did not find an essential role for EED in developmental angiogenesis. These studies may point out differences in the dependence of developmental versus tumor angiogenesis on PRC2 or they may further highlight non-canonical roles of EZH2. Alternatively, technical differences in the method of gene inactivation may have led to divergent conclusions. A genetic deletion of Eed or Ezh2 by inducible CDH5 ${ }^{\text {CreETR2 }}$ in the adult mouse would help to solidify our current understanding of the role of PRC2 in angiogenesis and even possibly generate new insights into the functions of EED and EZH2 within PRC2 and in non-canonical roles. ${ }^{33}$

PRC2 and hematopoietic malignancies. Mutations of genes encoding PRC2 subunits have been linked to multiple types of human hematopoietic malignancies. Somatic mutation of EED or chromosome deletion of $E Z H 2$ led to myelodysplastic syndrome and related neoplasm, which is consistent to our and others studies that depletion of EZH2 and EED in hematopoietic cells impaired erythroblast generation, erythrocyte maturation, and caused cytopenia. ${ }^{31,32,38}$ Moreover, structural rearrange of EZH2, SUZ12, and EED both in mouse and human results in the T-cell acute lymphoblast leukemia. ${ }^{20}$ The malignant transformation in hematopoietic cells lacking PRC2 suggests that compensating for PRC2 deficiency may be a productive therapeutic strategy for some hematopoietic disorders. Consistent with this idea, overexpression of EZH2 in mice augmented HSC regeneration and prevented the HSC exhaustion during the transplantation assays. ${ }^{32}$ However, elevation of EZH2 and other PRC2 components is associated with aggressive forms in solid cancers and related to cancer progression. ${ }^{39,40}$ As a result, PRC2 inhibition has emerged as a potential strategy cancer treatment strategy, and is currently being tested in clinical trials. Given the deleterious effects of PRC2 inhibition on 

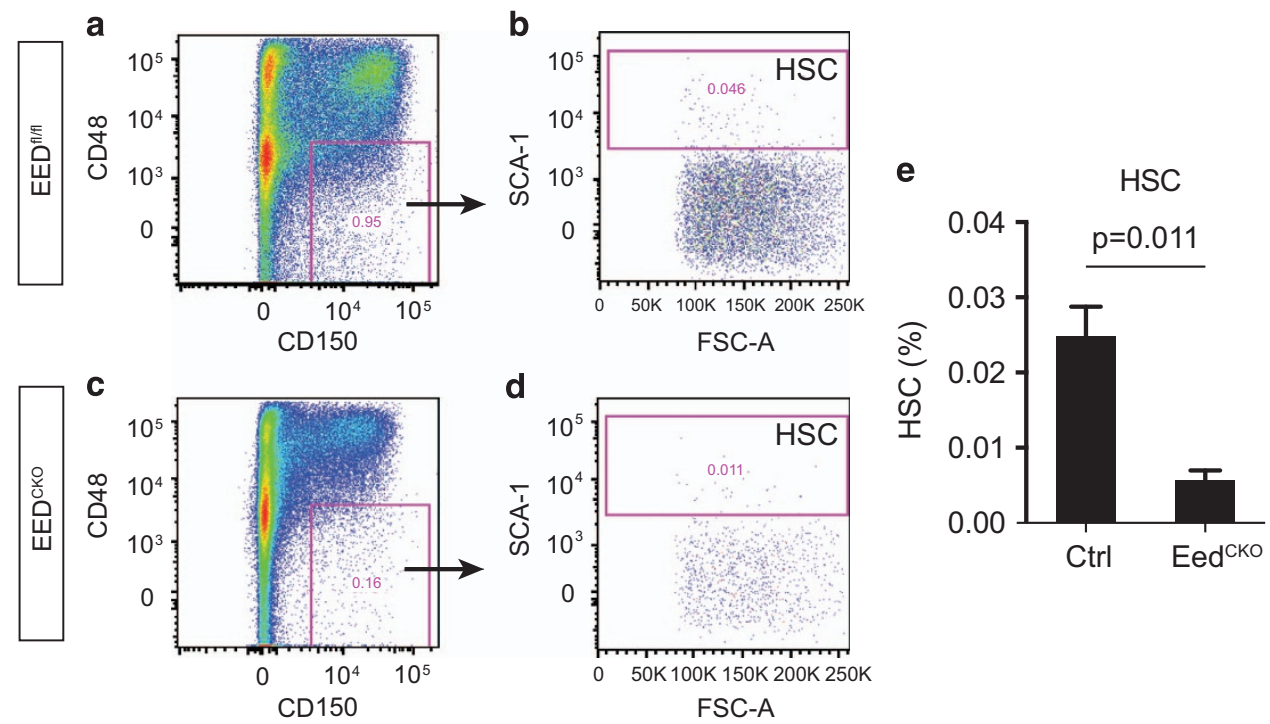

Figure 6 Eed deletion resulted in defective HSC development. (a-d) FACS strategy defining HSC cells in wild-type and EED ${ }^{\text {CKO }}$ mouse. (e) Quantitative analysis of HSC cells. Unpaired $t$-test, $n=3-5$

HSCs and hematopoiesis illuminated in this study and others, the side effects and therapeutic index of these agents needs to be carefully evaluated.

\section{Materials and Methods}

Mice. All animal procedures were approved by Institutional Animal Care and Use Committee (IACUC) of Shanghai Jiao Tong University and Boston Children's Hospital. The EED flox, Tie2Cre, and Rosa26fsYFP alleles were described previously. ${ }^{23,41,42}$ All mouse strains were maintained in a C57BL/6 and 129 mixed background. EED ${ }^{\mathrm{f} / \mathrm{fl}}$ female mice were mated with $\mathrm{Tie}^{\mathrm{Cre}}$;EED EED $^{\mathrm{fl} / \mathrm{at}}$ male mice to generate $\mathrm{Tie}^{\mathrm{Cre}} ; \mathrm{EED}^{\mathrm{fl} / \mathrm{fl}}$ embyros, to avoid germline deletion that can result from Tie2Cre transmitted through the female germline. Gestational age of embryos was determined by checking vaginal plugs, with noon of the day of the plug defined as embryonic day (E) E0.5.

Immunofluorescent staining. Immunofluorescent staining of embryo sections was performed as described previously. ${ }^{43,44}$ Cryopreserved embryos were sectioned, fixed in 4\% PFA for $15 \mathrm{~min}$, and blocked and permeabilized with Blocking buffer (1\% BSA in PBS containing 5\% goat serum) containing $0.1 \%$ Triton X-100. Following $1 \mathrm{~h}$ of blocking at room temperature, the sections were incubated with primary antibodies (H3K27me3, 17-622, Millipore; PECAM1, clone MEC13.3, BD Bioscience, San Diego, USA) overnight at $4{ }^{\circ} \mathrm{C}$. The samples were washed three times with PBS containing $0.05 \%$ Tween-20, incubated with Alexa-conjugated secondary antibodies (1:200, Fisher Scientific, Shanghai, China) for $1 \mathrm{~h}$ and then counterstained with DAPI ( $1 \mu \mathrm{g} / \mathrm{ml}$, Roche) for $5 \mathrm{~min}$ before mounting. The images were captured on a Olympus FluoView FV1000 confocal microscope (China).

Whole-mount staining. Whole-mount immunohistochemical staining of E9.5 and E12.5 mouse embryos using PECAM1 antibody was performed as reported previously. ${ }^{43,44}$ Briefly, embryos were dissected from the uterus, fixed in $4 \%$ paraformaldehyde overnight, and dehydrated in $100 \%$ methanol until use. The embryo was quenched with $5 \%$ hydrogen peroxide in methanol to remove the endogenous peroxidase and then rehydrated in 75,50 , and $25 \%$ methanol before use. The blocking process was performed with incubation of blocking buffer $(2 \%$ nonfat milk, $5 \%$ goat serum and $0.2 \%$ Triton $\mathrm{X}-100)$ for $2 \mathrm{~h}$ at room temperature. After blocking, PECAM1 antibody (MEC13.3, 1:200; BD Pharmingen) in blocking buffer was applied to embryos with gentle shaking overnight. The embryos were washed with PBS five times, incubated overnight with biotinylated goal anti-rat antibody (1:50, BD Pharmingen) in PBS containing $0.2 \%$ Triton X-100, and then incubated with HRP-conjugated streptavidin for $2 \mathrm{~h}$ before color development in DAB substrates (Vector Labs, Burlingame, CA, USA). The images were obtained using an SMZ800 stereo microscope.
Flow cytometry. Fetal livers were isolated from E12.5 mouse embryos, triturated, and passed through $70 \mu \mathrm{m}$ nylon mesh to obtain a single-cell suspension. The RBCs were lysed in $1 x$ erythroid lysis buffer. The isolated cells were first incubated on ice with Fc-Block. For selection of Lin ${ }^{-}$cells, the cells were incubated with biotin-conjugated antibodies against lineage-specific markers (Ter119[TER119]; CD71; B220[RA3-6B2]; Gr1[RB6-8C5]), and biotin was subsequently bound by PerCP-Cy5.5 conjugated streptavidin. The cells were stained as indicated for surface markers, including FITC-CD34, phycoerythrin(PE)-Cy7-conjugated Sca1 (D7), allophycocyanin (APC)-Cy7-conjugated cKit (2B8), and PE-conjugated CD48 (HM48-1). These antibodies were purchased from BD Biosciences (San Jose, CA, USA), Thermo Fisher (Shanghai, China) and BioLegend (San Diego, CA, USA). The dead cells were excluded by staining with 7-AAD ( $1 \mu \mathrm{g} / \mathrm{ml}$, Thermo Fisher). Antibody-labeled cells were run on an LSR II/LSR Fortessa for analysis or on a FACSAria II for cell sorting. Flowjo software (Ashland, OR, USA) was used to analyze the cytometry data.

Statistics. The results are shown as mean \pm S.E.M. Student's $t$-test was used to determine whether groups were significantly different.

\section{Conflict of Interest}

The authors declare no conflict of interest.

Acknowledgements. WTP was supported by an AHA Established Investigator Award and NIH U01HL098166. BZ was funded by National Science Foundation of China (91539109 and 31671503), A Thousand Young Talent Award (16Z127060017), NIH T32 HL7572-28, and an AHA Scientist Development Grant 14SDG20380866.

\section{Author contributions}

BZ conceived of the study. WY, FZ, BZ and WTP designed the experiments. WY, SW and YF performed the bench work. WY, FZ, JC and BZ performed the data analysis. WY, WTP and BZ drafted the manuscript. All the authors read and revised the paper.

1. Margueron R, Reinberg D. The Polycomb complex PRC2 and its mark in life. Nature 2011; 469: 343-349.

2. Whitcomb $\mathrm{SJ}$, Basu $A, A$ Allis $C D$, Bernstein E. Polycomb Group proteins: an evolutionary perspective. Trends Genet 2007; 23: 494-502.

3. Margueron R, Li G, Sarma K, Li G, Sarma K, Blais A et al. Ezh1 and Ezh2 maintain repressive chromatin through different mechanisms. Mol Cell 2008; 32: 503-518.

4. Shen X, Liu Y, Hsu YJ, Liu Y, Hsu YJ, Fujiwara Y et al. EZH1 mediates methylation on histone $\mathrm{H} 3$ lysine 27 and complements EZH2 in maintaining stem cell identity and executing pluripotency. Mol Cell 2008; 32: 491-502. 
5. Cao R, Wang L, Wang $H$, Wang $L$, Wang $H$, Xia $L$ et al. Role of histone $H 3$ lysine 27 methylation in Polycomb-group silencing. Science 2002; 298: 1039-1043.

6. Kuzmichev A, Nishioka K, Erdjument-Bromage $H$, Tempst $P$, Reinberg D. Histone methyltransferase activity associated with a human multiprotein complex containing the Enhancer of Zeste protein. Genes Dev 2002; 16: 2893-2905.

7. Faust $\mathrm{C}$, Schumacher A, Holdener B, Magnuson T. The eed mutation disrupts anterior mesoderm production in mice. Development 1995; 121: 273-285.

8. Faust C, Lawson KA, Schork NJ, Thiel B, Magnuson T. The Polycomb-group gene eed is required for normal morphogenetic movements during gastrulation in the mouse embryo. Development 1998; 125: 4495-4506.

9. Tee WW, Reinberg D. Chromatin features and the epigenetic regulation of pluripotency states in ESCs. Development 2014; 141: 2376-2390.

10. Ezhkova E, Pasolli HA, Parker JS, Pasolli HA, Parker JS, Stokes N et al. Ezh2 orchestrates gene expression for the stepwise differentiation of tissue-specific stem cells. Cell 2009; 136 $1122-1135$.

11. Ezhkova E, Lien WH, Stokes N, Pasolli HA, Silva JM, Fuchs E. EZH1 and EZH2 cogovern histone H3K27 trimethylation and are essential for hair follicle homeostasis and wound repair. Genes Dev 2011; 25: 485-498.

12. $\mathrm{He} A, \mathrm{Ma} Q, \mathrm{Cao} J, \mathrm{Ma} Q, \mathrm{Cao} J$, von Gise $\mathrm{A}$ et al. Polycomb repressive complex 2 regulates normal development of the mouse heart. Circ Res 2012; 110: 406-415.

13. Delgado-Olguin P, Huang Y, Li X, Christodoulou D, Seidman CE, Seidman JG et al. Epigenetic repression of cardiac progenitor gene expression by Ezh2 is required for postnatal cardiac homeostasis. Nat Genet 2012; 44: 343-347.

14. Lu C, Han HD, Mangala LS, Ali-Fehmi R, Newton CS, Ozbun L et al. Regulation of tumor angiogenesis by EZH2. Cancer Cell 2010; 18: 185-197.

15. Orkin SH, Zon LI. Hematopoiesis: an evolving paradigm for stem cell biology. Cell 2008; 132 631-644.

16. Akashi K, Traver D, Kondo M, Weissman IL. Lymphoid development from hematopoietic stem cells. Int J Hematol 1999; 69: 217-226.

17. Iwasaki H, Akashi K. Myeloid lineage commitment from the hematopoietic stem cell. Immunity 2007; 26: 726-740.

18. Ueda T, Sanada M, Matsui H, Yamasaki N, Honda Zl, Shih LY et al. EED mutants impai polycomb repressive complex 2 in myelodysplastic syndrome and related neoplasms.[letter]. Leukemia 2012; 26: 2557-2560.

19. Stepanik VA, Harte PJ. A mutation in the $E(Z)$ methyltransferase that increases trimethylation of histone $\mathrm{H} 3$ lysine 27 and causes inappropriate silencing of active Polycomb target genes. Dev Biol 2012; 364: 249-258.

20. Simon C, Chagraoui J, KrosI J, Gendron P, Wilhelm B, Lemieux S et al. A key role for EZH2 and associated genes in mouse and human adult T-cell acute leukemia. Genes Dev 2012; 26: $651-656$.

21. Sauvageau M, Sauvageau G. Polycomb group proteins: multi-faceted regulators of somatic stem cells and cancer. Cell Stem Cell 2010; 7: 299-313.

22. Mochizuki-Kashio M, Mishima $Y$, Miyagi S, Negishi M, Saraya A, Konuma $T$ et al. Dependency on the polycomb gene Ezh2 distinguishes fetal from adult hematopoietic stem cells. Blood 2011; 118: 6553-6561.

23. Xie H, Xu J, Hsu JH, Nguyen M, Fujiwara Y, Peng $\mathrm{C}$ et al. Polycomb repressive complex 2 regulates normal hematopoietic stem cell function in a developmental-stage-specific ?thyc?> manner. Cell Stem Cell 2014; 14: 68-80.

24. Lessard J, Schumacher A, Thorsteinsdottir U, van Lohuizen M, Magnuson T, Sauvageau G Functional antagonism of the Polycomb-Group genes eed and Bmi1 in hemopoietic cell proliferation. Genes Dev 1999; 13: 2691-2703.

25. Rivera-Feliciano J, Lee KH, Kong SW, Rajagopal S, Ma Q, Springer Z et al. Development of heart valves requires Gata4 expression in endothelial-derived cells. Development 2006; 133 3607-3618.

26. Zhang H, von Gise A, Liu Q, Hu T, Tian X, He L et al. Yap1 is required for endothelial to mesenchymal transition of the atrioventricular cushion. J Biol Chem 2014; 289 18681-18692.

27. Liakhovitskaia A, Gribi R, Stamateris E, Villain G, Jaffredo $T$, Wilkie $R$ et al. Restoration of Runx1 expression in the Tie2 cell compartment rescues definitive hematopoietic stem cells and extends life of Runx1 knockout animals until birth. Stem Cells 2009; 27: 1616-1624.
28. Palis J, Robertson S, Kennedy M, Wall C, Keller G. Development of erythroid and myeloid progenitors in the yolk sac and embryo proper of the mouse. Development 1999; 126 : 5073-5084.

29. Arinobu Y, Iwasaki H, Gurish MF, Mizuno S, Shigematsu H, Ozawa H et al. Developmental checkpoints of the basophil/mast cell lineages in adult murine hematopoiesis. Proc Natl Acad Sci USA 2005; 102: 18105-18110.

30. Kamminga LM, Bystrykh LV, de Boer A, Houwer S, Douma J, Weersing E et al. The Polycomb group gene Ezh2 prevents hematopoietic stem cell exhaustion. Blood 2006; 107: 2170-2179.

31. Makishima H, Jankowska AM, Tiu RV, Szpurka H, Sugimoto Y, Hu Z et al. Novel homo- and hemizygous mutations in EZH2 in myeloid malignancies.[letter]. Leukemia 2010; 24: 1799-1804.

32. Herrera-Merchan A, Arranz L, Ligos JM, de Molina A, Dominguez O, Gonzalez S. Ectopic expression of the histone methyltransferase Ezh2 in haematopoietic stem cells causes myeloproliferative disease. Nat Commun 2012; 3: 623.

33. Xu K, Wu ZJ, Groner AC, He HH, Cai C, Lis RT et al. EZH2 oncogenic activity in castrationresistant prostate cancer cells is Polycomb-independent. Science 2012; 338: 1465-1469.

34. Cao Q, Wang X, Zhao M, Yang R, Malik R, Qiao Y et al. The central role of EED in the orchestration of polycomb group complexes. Nat Commun 2014; 5: 3127

35. Rafii S, Butler JM, Ding BS. Angiocrine functions of organ-specific endothelial cells. Nature 2016; 529: 316-325.

36. Pitulescu ME, Schmidt I, Benedito R, Adams RH. Inducible gene targeting in the neonatal vasculature and analysis of retinal angiogenesis in mice. Nat Protoc 2010; 5: 1518-1534.

37. Delgado-Olguín P, Dang LT, He D, Thomas S, Chi L, Sukonnik T et al. Ezh2-mediated repression of a transcriptional pathway upstream of Mmp9 maintains integrity of the developing vasculature. Development 2014; 141: 4610-4617.

38. Morin RD, Johnson NA, Severson TM, Mungall AJ, An J, Goya R et al. Somatic mutations altering EZH2 (Tyr641) in follicular and diffuse large B-cell lymphomas of germinalcenter origin. Nat Genet 2010; 42: 181-185

39. Lee ST, Li Z, Wu Z, Aau M, Guan P, Karuturi RK et al. Context-specific regulation of NF-кB target gene expression by EZH2 in breast cancers. Mol Cell 2011; 43: 798-810.

40. Varambally S, Dhanasekaran SM, Zhou M, Barrette TR, Kumar-Sinha C, Sanda MG et al. The polycomb group protein EZH2 is involved in progression of prostate cancer. Nature 2002; 419: 624-629.

41. Srinivas S, Watanabe T, Lin CS, William CM, Tanabe Y, Jessell TM et al. Cre reporter strains produced by targeted insertion of EYFP and ECFP into the ROSA26 locus. BMC. Dev Biol 2001; $1: 4$

42. Kisanuki YY, Hammer RE, Miyazaki J, Williams SC, Richardson JA, Yanagisawa M. Tie2-Cre transgenic mice: a new model for endothelial cell-lineage analysis in vivo. Dev Biol 2001; 230: 230-242.

43. Zhang B, Xiao W, Qiu H, Zhang F, Moniz HA, Jaworski A et al. Heparan sulfate deficiency disrupts developmental angiogenesis and causes congenital diaphragmatic hernia. J Clin Invest 2014; 124: 209-221.

44. Zhang B, Dietrich UM, Geng JG, Bicknell R, Esko JD, Wang L. Repulsive axon guidance molecule Slit3 is a novel angiogenic factor. Blood 2009; 114: 4300-4309.

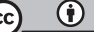

Cell Death and Disease is an open-access journal published by Nature Publishing Group. This work is licensed under a Creative Commons Attribution 4.0 International License. The images or other third party material in this article are included in the article's Creative Commons license, unless indicated otherwise in the credit line; if the material is not included under the Creative Commons license, users will need to obtain permission from the license holder to reproduce the material. To view a copy of this license, visit http://creativecommons.org/licenses/by/4.0/

(C) The Author(s) 2017 